\title{
CONSUMO DE DROGAS DE USO LÍCITO E ILíCITO EN JÓVENES UNIVERSITARIOS DE LA U.D.C.A
}

\section{ILLICIT AND LICIT DRUG CONSUMPTION BY U.D.C.A'S COLLEGE STUDENTS}

\author{
Linda Shirley Segura ${ }^{1}$, Nelly Esther Cáliz ${ }^{2}$ \\ ${ }^{1}$ Enfermera. Universidad de Ciencias Aplicadas y Ambientales U.D.C.A, calle 6A este \# 5-03, Facatativá, Cundinamarca, \\ e-mail: lisegura@udca.edu.co; ${ }^{2}$ Enfermera, Magister en Psicología Comunitaria, Candidata a Doctora en Salud Colectiva, \\ Docente titular. Universidad de Ciencias Aplicadas y Ambientales U.D.C.A, calle 222 No. 55-30, Bogotá, Colombia, e-mail: \\ ncaliz@udca.edu.co
}

Rev. U.D.C.A Act. \& Div. Cient. 18(2): 311-319, Julio-Diciembre, 2015

\section{RESUMEN}

Se investigó el consumo de drogas de uso lícito e ilícito en jóvenes universitarios de la U.D.C.A, bajo un diseño descriptivo, observacional y de corte transversal, en una muestra de 128 estudiantes. El instrumento utilizado fue el cuestionario, aplicado en el II estudio epidemiológico andino sobre consumo de drogas en la población universitaria, del 2012 y el Test de trastornos relacionados con el consumo de alcohol (AUDIT). Los principales resultados muestran que las sustancias más consumidas son el alcohol, el tabaco y la marihuana; los determinantes sociales del consumo, se asocian a la facilidad de acceso, a la oferta y oportunidad de probar drogas de uso ilícito, al igual que la curiosidad y el consumo de este tipo de sustancias, por parte de amigos. Como factor protector, se identificó el decir "No" ante la oportunidad de consumo. Se concluye y se recomienda la participación activa de la Universidad, en programas promocionales de la salud, que se fundamenten en la constitución de un joven crítico y autónomo frente a la oferta y consumo de sustancias psicoactivas.

Palabras clave: Drogas ilícitas, adolescente, determinantes sociales de la Salud, universidades, consumidores de drogas.

\section{SUMMARY}

The consumption of licit and illicit drug use among university students of U.D.C A, under a descriptive, observational and cross-sectional design, in a sample of 128 students was investigated. The instrument used was the questionnaire applied in the II Andean epidemiological study on drug use in the student population of 2012 and the Test of disorders related to alcohol consumption (AUDIT). The main results show that the most consumed substances are alcohol, snuff and marijuana; social determinants of consumption are associated with the accessibility, supply and opportunity to test drugs for illicit use, like curiosity and the consumption of these substances by friends. As a protective factor saying "No" to the consumer opportunity was identified. The active participation of the University in health promotion programs, based on the formation of a critical and independent young facing the supply and consumption of psychoactive substances is recommended

Key words: Illicit drugs, teenager, social determinants of health, universities, drug users.

\section{INTRODUCCIÓN}

El consumo de sustancias psicoactivas ha sido una práctica cultural y ancestral desde los inicios de la humanidad y es así como comunidades antiguas del medio oriente, Asia, América, Europa, África y comunidades indígenas actuales, utilizaron y utilizan estas sustancias al interior de su medicina tradicional o en las experiencias rituales, como medio para transitar al plano espiritual, sin que ello represente un problema social (Sierra, 2012); sin embargo, en sociedades occidentalizadas modernas, esta práctica ha alcanzado dimensiones sociales totalmente diferentes y el uso de drogas, en el ámbito mundial y en poblaciones de países con serios problemas sociopolíticos y económicos, como Colombia, El Salvador, Perú, Ecuador, México y, en general, América Latina, se constituye en un problema, que afecta a individuos, familias y comunidades, tanto por la adicción física y psíquica del consumidor problemático y su exposición a enfermedades como el SIDA y la hepatitis B, como por los efectos sociales del narco y el microtráfico de estupefacientes, sobre la sociedad. 
El consumo de sustancias psicoactivas, como un fenómeno social que, además de estar determinado en el plano singular por las características del sujeto consumidor y su relación con la sustancia psicoactiva, es necesario abordar. También, es un evento que emerge en el plano particular, como resultado de las condiciones de vida de las personas y del microtráfico en el territorio y en el plano general, por aspectos, como la alta rentabilidad económica del narcotráfico y las prácticas corruptivas de estamentos gubernamentales y no gubernamentales, involucrados con las mafias y la economía criminal.

Con respecto al plano general, los informes presentados por la ONU contra la Droga y el Delito muestran que, a nivel mundial, el tamaño total de los mercados de las drogas de uso ilícito pueden llegar a unos US\$320.000 millones, lo que equivale el 0,9\% del PIB mundial; por su parte, los mercados de venta minorista de drogas en las Américas fueron estimados en $\$ 151.000$ millones, es decir, aproximadamente, el $47 \%$ del total mundial, en los cuales, Norteamérica participa alrededor del $44 \%$ del total mundial y Europa, con el 33\%; en cambio, el mercado de Sudamérica, Centroamérica y el Caribe fue cerca del 3\%, del total mundial (OEA, 2013). En esta dinámica, Colombia ingresa, como parte de las redes de narcotráfico, hacia finales de la década de 1960, inicialmente, con la marihuana y, hoy, es reconocido como el segundo productor de cocaína en el mundo (El Tiempo, 2014).

Como ya se mencionó, el problema social en torno a la producción, la distribución y el consumo de sustancias psicoactivas emergen según el contexto político, económico y cultural del país y es así como en Colombia, cuatro situaciones determinaron, en términos generales, el fenómeno del narcotráfico, en torno a la cocaína, específicamente. El primero tiene relación con el poder latifundista, que obliga al campesino a emigrar hacia terrenos baldíos, por fuera de la frontera agrícola y el cultivo de la hoja de coca, que proviene de Bolivia, se convierte en una opción económica para su sobrevivencia (Tascon, 2012; Thoumi, 2009).

Segundo, la importación desregulada de alimento con la llegada del neoliberalismo, generó crisis económicas en muchos campesinos, que no tuvieron otra salida que complementar el cultivo de productos lícitos con cultivos ilícitos, como una manera de afrontarla; tercero, el financiamiento de la guerra civil no declarada, proveniente de la producción y de la comercialización de la cocaína por parte de los actores involucrados: guerrilla y paramilitarismo, que instaló, en el gran parte del territorio colombiano, el cultivo de la hoja de coca, por parte de familias campesinas y su venta a los narcos compradores, como la guerrilla y los paramilitares (Tascon, 2012; Thoumi, 2009). Los mismos autores indican, que las prácticas corruptivas del Estado, como cuarto factor categórico, permitieron crear todas las condiciones para que se afianzara la economía ilegal entorno a la producción y a la comercialización de algunos estupefacientes.

Estos cuatro aspectos constituyeron en Colombia, los principales factores determinantes, para consolidar la industria de la drogas y una fuerte economía ilegal, que no solo quedo circunscrita al campo económico, sino que, también, permeo el campo político y cultural, acuñando términos, como la narco política y la narco cultura (Mejía, 2010).

En el plano particular, dimensión relacionada con las condiciones y el modo de vida de los grupos sociales, los estudios identifican cuatro determinantes del consumo de drogas en los jóvenes: el primero de ellos hace referencia a la oferta y a la accesibilidad de drogas en el territorio, estimulada por el auge del microtráfico de sustancias psicoactivas, en aquellos lugares donde los jóvenes estudian, habitan, se divierten y circulan, como son los colegios, las universidades, la cercanía a los centros comerciales, los parques y los centros de diversión (Morales et al. 2011). Un segundo determinante corresponde al entorno familiar, en el que las sustancias psicoactivas hacen parte de las prácticas y de los hábitos de consumo o, en algunos casos, la venta de estas sustancias, como estrategia de sobrevivencia, a la que se dedican las mujeres con un limitado acceso al trabajo "legal", a las oportunidades sociales, económicas y culturales, para satisfacer sus necesidades y las de sus familias (Palma Campos, 2011).

Se identifican, también, las relaciones entre pares, como un determinante que, en este plano, puede incidir en el consumo o no de sustancias psicoactivas; al respecto, la literatura científica muestra que el uso de estas sustancias por parte de amigos y de compañeros de estudio, resulta ser un desencadenante de consumo en el joven (Varela et al. 2007; Londoño Pérez \& Valencia Lara, 2008; Rodríguez et al. 2012). Por último, en el plano particular de las condiciones de vida, las prácticas culturales -en torno al ocio y la recreación- son facilitadoras del uso de las sustancias psicoactivas, por parte de jóvenes universitarios. En la actualidad, el consumo recreativo de drogas, legales e ilegales, se ha convertido en una práctica común de muchos jóvenes, tal como lo muestra la revisión adelantada por Tomás et al. (2003), en el artículo, Jóvenes, fin de semana y uso recreativo de drogas: evolución y tendencias del ocio juvenil.

Otras investigaciones han identificado que las características personales, ubicados en el plano singular, que pueden predisponer al consumo o abuso de sustancias psicoactivas de uso legal e ilegal, por parte de la población universitaria, son la corta edad, la necesidad e importancia de sentirse aceptado, el poco interés en los riesgos del consumo y, en algunos casos, la ansiedad y la depresión; en esta época, se presenta una mayor vulnerabilidad a caer en un consumo irresponsable, por la facilidad de acceso al consumo recrea- 
tivo, que puede conllevar a los efectos más nocivos, como la dependencia, conductas sexuales riesgosas, abandono de las actividades académicas o problemas en las relaciones sociales (Prieto Silva et al. 2012).

Otro aspecto importante en el análisis del consumo de sustancias psicoactivas compete a la necesidad de caracterizar el sujeto que consume, la sustancia y el tipo de consumo. Para la población de jóvenes universitarios, específicamente en Latinoamérica, la sustancia psicoactiva más consumida de uso legal es el alcohol y de uso ilegal, la marihuana. En Colombia, el 95,8\% de los estudiantes declararon haber consumido alcohol alguna vez, seguido de Ecuador, con un $88,7 \%$; Perú, con $87,5 \%$ y Bolivia, un $77,1 \%$. La edad promedio de inicio del consumo de alcohol es de 17,5 años, en Bolivia; 15,3, en Colombia; 16,4, en Ecuador y 16,2 años, en Perú. Entre los consumidores de alcohol, del último año, un $39,7 \%$ de los estudiantes, de Bolivia; un $36,6 \%$, en Ecuador; un $31,1 \%$, en Colombia y un $25,1 \%$, en Perú; califica como consumidor que presenta riesgo o uso perjudicial de alcohol, siendo el mayor porcentaje entre los hombres que entre las mujeres, en todos los países (Secretaría General de la Comunidad Andina, 2012).

Respecto a la marihuana, 1 de cada 3 estudiantes, en Colombia, declara haber consumido esta sustancia, por lo menos una vez, mientras que en Ecuador, el 21,3\% y menos del $12 \%$, en Bolivia y en Perú, con una edad promedio de inicio de 18 años, presentándose mayor consumo en hombres que en mujeres, en los cuatro países. La prevalencia del último año de consumo de marihuana presenta tasas de 3,6\%, en Bolivia; 15,2\%, en Colombia; 8,8\%, en Ecuador y 4,2\%, en Perú. Es así como Colombia refleja la mayor tasa de consumo y, a la vez, los estudiantes colombianos manifiestan la menor percepción de riesgo, frente al uso de esta sustancia. Se estima, también, que entre un 26,0 y $29,2 \%$ de los estudiantes de Colombia, Ecuador y Perú pueden ser considerados abusadores o con signos de dependencia a la marihuana (Secretaría General de la Comunidad Andina, 2012).

Este panorama nos muestra que la práctica de consumo de sustancias psicoactivas es una situación de alcance local y mundial, siendo realmente problemática las estadísticas que indican signos de abuso, de dependencia y de muerte, en razón, primero, a que son seres humanos y, segundo, a un momento de vida, en el que hombres y las mujeres proyecta su existencia.

Estos signos de abuso o de dependencia son resultado del tipo de relación que se establezca con las sustancias, también denominado tipología del consumo, por la literatura científica. A la fecha, se han tipificado seis modalidades de consumo, entre ellos, el experimental, el recreativo, el habitual, el compulsivo, el problemático y, por último, el consumidor expendedor. El consumidor experimental es el individuo que prueba una o más sustancias, una o dos veces y decide no volverlo a hacer; el recreativo, cuando es realizado con mediana regularidad entre círculos de amigos, en contextos de ocio (rumba, paseos), pero también ocupa su tiempo libre en otras actividades, donde no hay presencia de sustancias psicoactivas y no lo considera como un "escape" o una "solución" a sus problemas; el habitual, donde la sustancia psicoactiva ya juega un papel importante en la vida y cumple funciones cada vez que la consume, por lo que es muy probable que lo haga repetidamente; el compulsivo, se evidencian signos de dependencia, no sólo de la sustancia psicoactiva sino, adicionalmente, de los amigos y del estilo de vida que desarrolla en torno a ella; consumo problemático, se_evidencian problemas sobre la salud física, mental y social y, finalmente, el consumidor-expendedor, referido a las personas que comercializan pequeñas dosis de sustancias psicoactivas ilegales, con la intención de financiar su propio consumo, que se encuentra en las categorías de problemático y de dependencia, antes expuestas (Secretaría Distrital de Salud, 2011).

Para el caso de la Universidad de Ciencias Aplicadas y Ambientales U.D.C.A, escenario de la presente investigación, existe por parte de docentes y de directivas, la percepción de un incremento en el consumo de sustancias psicoactivas, pues se han identificado lugares de expendio y de consumo al interior de campus. Investigaciones anteriores han mostrado que el consumo de sustancias psicoactivas de uso legal e ilegal es una práctica por parte de los estudiantes de la U.D.C.A; sin embargo, a la fecha no se había realizado un perfil de consumo y tampoco una sistematización de las aproximaciones iniciales, que la Dirección de Bienestar Social Universitario y el Comité de Ética Institucional, tenían con respecto a esta problemática.

Es a partir de este contexto, donde surge la pregunta que guio este trabajo: ¿Cuál es el consumo de drogas de uso lícito e ilícito en los jóvenes universitarios de las áreas de Ciencias de la Salud y Ciencias Pecuarias de la Universidad de Ciencias Aplicadas y Ambientales U.D.C.A, año 2014? y, en consecuencia, el objetivo general asociado al establecimiento de dicho perfil.

\section{MATERIALES Y MÉTODOS}

La investigación, se desarrolló bajo un enfoque cuantitativo de tipo observacional, descriptivo y transversal. La población de estudio fueron 2.096 estudiantes, matriculados para el primer semestre de 2014, en los programas académicos de Enfermería, Medicina Humana, Medicina Veterinaria y Medicina Veterinaria y Zootecnia. La muestra correspondió a 128 jóvenes universitarios, seleccionados mediante muestreo probabilístico, estratificado y se calculó con la fórmula para 
poblaciones finitas, tomada de Briones (2003). Para seleccionar la población participante, se aplicaron los siguientes criterios de inclusión: estudiantes de los programas académicos establecidos para la muestra, hombres, mujeres, de segundo a último semestre y que respondieron sí, en el consentimiento informado.

Instrumentos de investigación: El instrumento utilizado fue el mismo aplicado en el II estudio epidemiológico Andino sobre consumo de drogas en la población universitaria de 2012, elaborado por el Observatorio Interamericano sobre Drogas (OID), Comisión Interamericana para el Control del Abuso de Drogas (CICAD) y profesionales del Programa Antidrogas llícitas de la Comunidad Andina (PRADICAN), validado para la población universitaria en Colombia, que puede ser consultado en: http://cicad.oas.org/oid/pubs/PRADICAN_Informe_Regional.pdf. Para evaluar la tasa de abuso del alcohol, se aplicó un instrumento de percepción, a aquellas personas que declararon haber consumido esta sustancia en los últimos doce meses; este instrumento es el AUDIT (Babor et al. 2001), sugerido por la Organización Mundial de la Salud, que evalúa el uso perjudicial o riesgoso del alcohol.

Trabajo de campo: El proceso de investigación, se llevó a cabo en tres momentos: el primero, una revisión exhaustiva, en artículos científicos originales, de revisión y reflexión, sobre el fenómeno de estudio y el consumo de drogas en jóvenes universitarios; el segundo, la búsqueda y la selección de un instrumento validado para la recolección de información y, el tercer momento, el trabajo de campo; por último, se procesó la información y se elaboró el análisis estadístico descriptivo, en una base de datos, creada en Microsoft Excel.
Consideraciones éticas: Cumpliendo con los criterios éticos de la investigación en salud, se explicó y se entregó el consentimiento informado, con capacidad de libre elección y sin coacción alguna a los participantes, al inicio de los grupos focales, basado en la Resolución No 008430 de 1993.

\section{RESULTADOS Y DISCUSIÓN}

Caracterización de las sustancias, los participantes y el tipo consumo: Las sustancias psicoactivas de uso legal más consumidas por la población encuestada son el tabaco, el alcohol y las drogas farmacológicas, como los tranquilizantes y, de uso ilegal, la marihuana (Gráfico 1), comportamiento similar a las estadísticas registradas en los últimos Informes Mundiales sobre Drogas (UNODC, 2012; 2013), en el Estudio Nacional de Consumo de Sustancias Psicoactivas en Colombia, de 2013 (Gobierno Nacional de la Republica de Colombia, 2013), en el II Estudio Epidemiológico Andino sobre Consumo de Drogas en la Población Universitaria (Secretaría General de la Comunidad Andina, 2012) y en otras investigaciones, como la adelantada en Brasil, por Ortega-Pérez et al. (2011), con una muestra de 500 jóvenes universitarios.

Para el caso del alcohol, que es la droga más consumida en la población participante, los indicadores de uso en este estudio (Gráfico 1) son similares al II Estudio Epidemiológico Andino, que reporta una prevalencia del 95\%, alguna vez en la vida; $85 \%$, último año y $61 \%$, último mes y aunque la diferencia entre el primer momento de consumo, con respecto al consumo actual, en los dos casos, sea aproximadamente del 30\%, la prevalencia sigue siendo alta. El alcohol es conocido como la droga de iniciación, seguida del tabaco y la

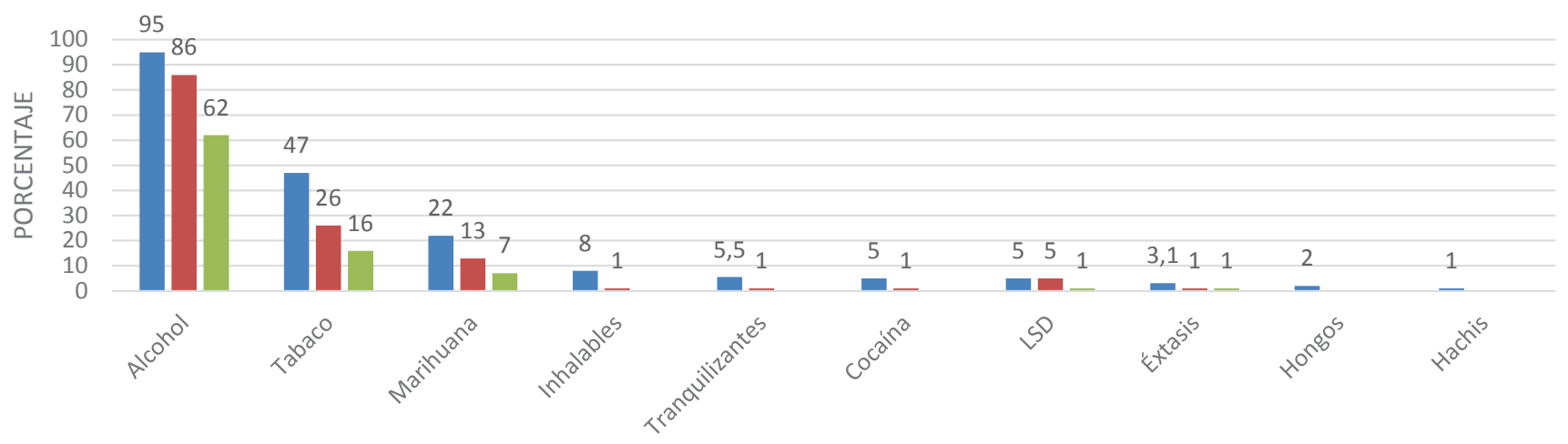

SUSTANCIA PSICOACTIVA

- PREVALENCIA DEL CONSUMO

- PREVALENCIA DEL CONSUMO

— PREVALENCIA DEL CONSUMO

Gráfico 1. Prevalencia de consumo de sustancias psicoactivas de uso legal e ilegal. 
marihuana, resultados similares a los reportados en los estudios de Ortega et al. (2011), Arrieta Vergara (2009), Medina et al. (2009), Sepúlveda et al. (2011) y Montoya et al. (2009).

En cuanto a los participantes, los hallazgos evidencian consumo en hombres y en mujeres de sustancias de uso legal, como el tabaco, el alcohol y los tranquilizantes; para el caso de la población LGBT, solo se observó consumo de alcohol. Por las diferencias que se reflejan en los diferentes momentos de consumo, alguna vez en la vida, reciente y actual, la proporción de consumidores habituales, compulsivos o problemáticos es menor a los consumidores experimentales y recreativos, lo que podría ser, un resultado del control que ejercen las normas sociales sobre los individuos, frente al uso de este tipo de sustancias, tal como se presenta en estudios realizados por Varela et al. (2007), en los cuales, la valoración negativa hacia las sustancias, tal como hacer el ridículo, comportarse indebidamente, involucrarse en situaciones peligrosas y llegar a la adicción, limitan, de manera importante, el consumo de las misma o también la capacidad de autocontrol y autorregulación, como lo han confirmado los estudios adelantados por Cáceres et al. (2006) y Calderón \& Cáliz (2015).

Resulta preocupante que de la población que indicó tener consumo reciente de alcohol, el 30,91\% presentan signos de dependencia, situación predominante en los programas de ciencias de la salud, grupo que, en teoría, maneja mayor información relacionada con factores de riesgos asociados a la salud. Este patrón de consumo es similar a un estudio realizado en población estudiantil, de una universidad pública en Medellín, en donde se halló que la totalidad de estudiantes muestran algún grado de riesgo en el consumo de alcohol, siendo elevada la proporción de estudiantes, con síntomas de dependencia (31\%) y con consumo perjudicial (7\%), lo que pone de manifiesto que, en la actual población, al igual que en todo el mundo, el uso y el abuso de alcohol es un problema de salud pública, que afecta a todos grupos etarios, particularmente, en adolescentes y en adultos jóvenes universitarios (Cardona \& Orjuela, 2014; Castaño \& Calderón, 2014).

Respecto al consumo de marihuana, se encontró que el 13\% de los estudiantes, quienes declararon tener consumo reciente para esta droga, el 30\% presentan signos de abuso; el $48 \%$, de dependencia y el $11 \%$ de los estudiantes tienen, tanto signos de abuso como de dependencia, que se clasificarían como consumidores compulsivos o problemáticos. Para el consumo de cocaína no se encontraron hallazgos al respecto.

Determinantes sociales que exponen a la población participante, al consumo de sustancias psicoactivas de uso legal e ilegal: En el estudio, se identificaron cuatro determinantes sociales, dos del plano particular, relacionadas con las condiciones de vida y dos del plano singular, asociados a las motivaciones de los jóvenes. Con respecto al plano particular, las variables estimadas fueron: facilidad de acceso, oferta y oportunidad de probar drogas de uso ilícito.

Los datos que se muestran en el gráfico 2, dejan entrever que la oferta de sustancias psicoactivas de uso ilegal, es una realidad para este grupo de jóvenes, a lo largo de su trayectoria universitaria y que, además, dicha oferta puede ser parte de la dinámica universitaria, a través de las redes de microtráfico, que pueden operar dentro y fuera de la universidad, tal como se muestran en el $20 \%$ de los encuestados, al manifestar que tiene conocimiento de lugares dentro de la Universidad, donde se venden drogas y, en el $16 \%$, que saben de lugares, cerca de la universidad, donde se pueden conseguir este tipo de sustancias.

Esta situación, se constituye en un determinante importante del inicio y del consumo de estas sustancias y, aunque pocas investigaciones resaltan la oferta y la accesibilidad, como un condicionante importante en el consumo, se hace pertinente la reflexión de Calafat (2007) cuando plantea que:

"Los hábitos de consumo no son precisamente una forma de resistencia o de construcción de la identidad juvenil autónoma, sino más bien el fruto de la presión de una poderosa presión económica y cultural que conduce a muchos jóvenes a estilos de vida evasivos o fragmentados por la compulsión. Detrás de todo ello están las alcoholeras, pero mucho más importante es la industria de la diversión, con un poder clave no sólo en el orden económico o de influencias políticas, sino en crear imaginario colectivo, en pensar que es lo que puede gustar a los adolescentes y jóvenes para que consuman más".

Con relación a este determinante, un factor que puede actuar como proceso protector frente a la exposición de los jóvenes a la oferta de sustancias psicoactivas ilícitas, hace referencia a la autonomía en la toma de decisiones y, según los datos para el grupo sujeto de estudio, el decir "No", es un hallazgo que pueda explicar el bajo consumo de sustancias de uso ilícitas. En este sentido, se exploró la relación entre la oportunidad en el uso de drogas y el consumo de marihuana, encontrando que los estadísticos descriptivos de chi cuadrado, arrojaron una relación significativa, tal como lo muestran las tablas 1 y 2 .

Por último, y en este mismo plano, cabe resaltar que aunque no se evidenció una relación estadísticamente significativa entre el consumo de sustancias de uso ilícito y aspectos asociados a satisfacción con la carrera, visión optimista del futuro, padres casados, buenas relaciones con ambos padres, mínimo de consumo de sustancias de uso ilícito en las familias y, finalmente, información recibida durante la etapa 


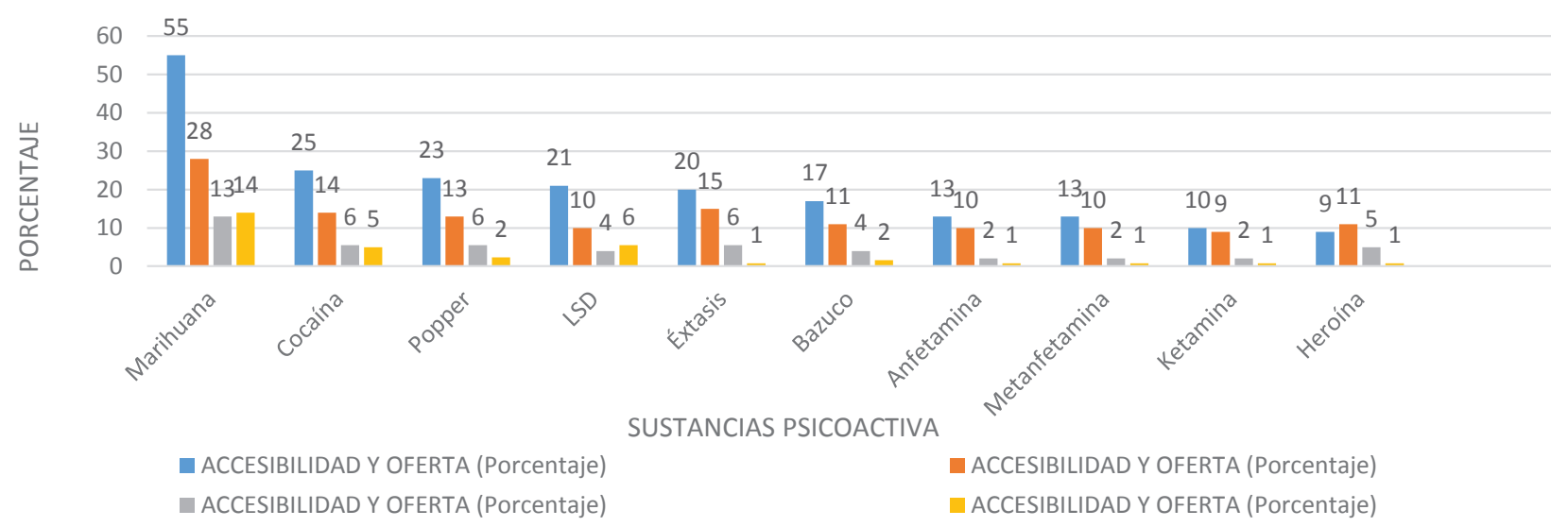

Gráfico 2. Accesibilidad y oferta de sustancias psicoactivas de uso ilegal.

Tabla 1. Pruebas de chi-cuadrado para la tabla de contingencia CONSUMO DE DROGAS ILÍCITAS [Marihuana] * Si tuviera la oportunidad, probaría alguna droga o sustancia ilícita.

\begin{tabular}{|l|r|c|c|}
\hline & Valor & Gl & Sig. asintótica (bilateral) \\
\hline $\begin{array}{l}\text { Chi-cuadrado de } \\
\text { Pearson }\end{array}$ & $16,659^{\mathrm{a}}$ & 6 &, 011 \\
\hline $\begin{array}{l}\text { Razón de } \\
\text { verosimilitudes }\end{array}$ & 18,872 & 6 &, 379 \\
\hline $\begin{array}{l}\text { Asociación lineal por } \\
\text { lineal }\end{array}$ &, 774 & 1 & \\
\hline No. de casos válidos & 128 & & 64 \\
\hline
\end{tabular}

a. 3 casillas $(25,0 \%)$ tienen una frecuencia esperada inferior a 5. La frecuencia mínima esperada es ,94.

escolar sobre prevención del uso de drogas, sí se resalta que la mayoría de estudiantes encuestados muestran indicadores positivos al respecto, como se señala en la gráficas 3 y 4 que, en investigaciones adelantadas por Rodríguez et al. (2012) y González et al. (2015 se comportaron como factores protectores frente al consumo de drogas.

En el plano singular, las variables estudiadas fueron percepción de riesgo y curiosidad frente al consumo, observando que no existe relación significativa entre la percepción del riesgo y el consumo, pero sí para las variables de curiosidad y consumo de drogas por parte de amigos, con un chi cuadrado menor a 0,05 , resultados que se ratifican en estudios adelantados por Calderón \& Cáliz (2015).

A manera de conclusión, los hallazgos de esta investigación confirman los resultados de otros estudios, en cuanto al tipo de sustancias y tipos de consumo, pero puede resultar novedoso en términos de los determinantes sociales del consumo, asociados a oferta, accesibilidad y oportunidad en el uso de las drogas, aspecto con poca evidencia en la literatura consultada.

Al respecto, una reflexión de cierre importante, en cuanto a considerar el consumo de drogas de uso legal e ilegal en jóvenes universitarios, como resultado de una red de determinaciones que transita en el plano general, del campo político y económico, como se mostró en la introducción de este artículo, aunque dichos aspectos no fueron objeto, ni alcance de estudio hacia el plano particular, es decir, su incidencia en el modo de vida y en el territorio donde habitan, estudian o trabajan las personas, ejemplo de ello, el microtráfico, la accesibilidad y la oferta de estupefacientes, las prácticas cul- 


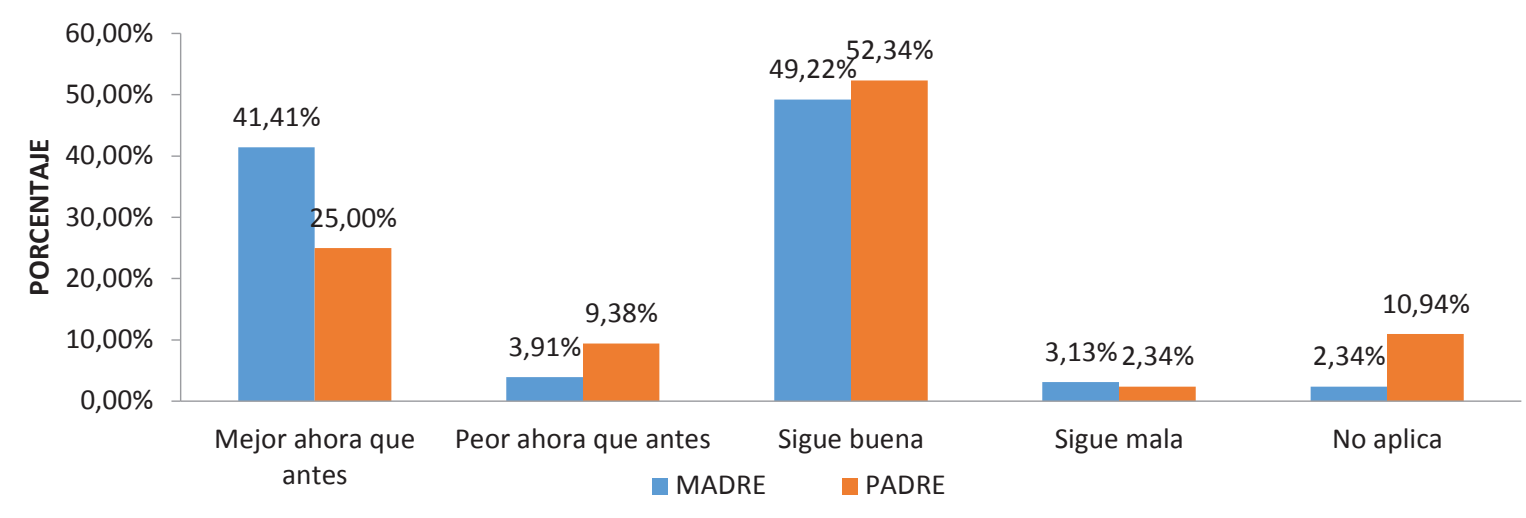

Gráfico 3. Relación con los padres.

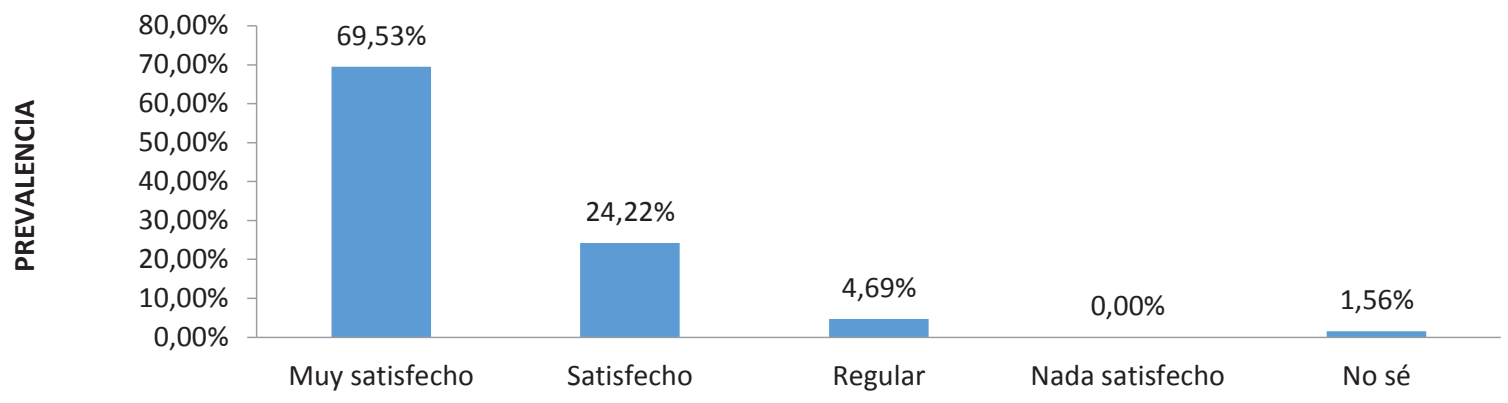

Gráfico 4. Satisfacción con la carrera.

turales frente al uso de psicoactivos de uso legal e ilegal en actividades de ocio y recreación, para materializarse -finalmente- en el consumo del joven universitario, quien puede disponer o no de capacidades para responder, de manera autónoma, ante la oferta de la sustancia o en la relación que establezca con ella, según haya sido su trayectoria de vida.

En esta línea de pensamiento, se recomienda que la Universidad sirva como un contexto abierto al razonamiento crítico frente al uso de la drogas lícitas e ilícitas, no solo en el marco de programas preventivos y asistenciales, sino, también, en la construcción y en la puesta en escena de estrategias colectivas, promocionales de la salud, en la que los(las) jóvenes -en espacios de debate y de reflexión-, asuman un papel protagónico en la construcción de una actitud y una práctica autónoma y consciente, frente al uso de las drogas.

Por ello, también se recomienda investigar, no solo en términos de procesos o factores deteriorantes que promueven el consumo, sino en procesos o factores protectores que posibilitan una práctica autónoma y una relación autorregulada con las sustancias psicoactivas, de las personas como sujetos individuales y colectivos, pues las prácticas de ocio y de recreación en la sociedad en general, son prácticas grupales.

Agradecimientos: Agradecemos a los estudiantes de la Universidad de Ciencias Aplicadas y Ambientales U.D.C.A por su actitud receptiva frente a la investigación y a los profesores y las directivas, por el apoyo en su implementación. Conflictos de intereses: El manuscrito fue preparado y revisado con la participación de todos los autores, quienes declaramos que no existe ningún conflicto de intereses que ponga en riesgo la validez de los resultados presentados. Financiación: Investigación adelantada en el marco del programa de la Vicerrectoría de Investigaciones, Joven investigador U.D.C.A. 


\section{BIBLIOGRAFÍA}

1. ARRIETA VERGARA, K. 2009. Consumo patológico de alcohol entre los estudiantes de la Universidad de Cartagena. Rev. Salud Pública, (Colombia). 11(6):878-886.

2. BABOR, T.; HIGGINS, J.; SAUNDERS, J.; MONTEIRO, M. 2001. Cuestionario de Identificación de los Trastornos debidos al Consumo de Alcohol. Organización Mundial de la Salud. Disponible desde Internet en: http://www.who.int/substance_abuse /activities/ en/AUDITmanualSpanish.pdf?ua $=1$ (con acceso $17 / 08 / 2015)$.

3. BRIONES, G. 2003. Métodos y técnicas de investigación para las ciencias sociales. Editorial Trillas. (México). 364p.

4. CÁCERES, D.; SALAZAR, I.; VARELA, M.; TOVAR, J. 2006. Consumo de drogas en jóvenes universitarios y su relación de riesgo y protección con los factores psicosociales. Univ. Psychol. 5(3):521-534.

5. CALAFAT, A. 2007. El abuso de alcohol de los jóvenes en España. Adicciones. 19(3):217-224.

6. CALDERÓN, E.A.; CÁLIZ, N.E. 2015. Juventud y universidad: sujetos y escenarios para el debate crítico y autorreflexivo sobre el consumo de sustancias psicoactivas de uso legal e ilegal. Gerencia y Políticas de Salud, 14(28). Disponible desde internet en: <http:// revistas.javeriana.edu.co/index.php/gerepolsal/article/view/13196/10538 (con acceso 02/10/2015).

7. CARDONA, J.; ORJUELA, L. 2014. Consumo de riesgo, síntomas de dependencia y consumo perjudicial de alcohol en estudiantes de una universidad pública de Medellín-2013. Disponible desde Internet en: dialnet. unirioja.es/descarga/articulo/5052064.pdf (con acceso 17/08/2015).

8. CASTAÑO, G.; CALDERÓN, G. 2014. Problemas asociados al consumo de alcohol en estudiantes universitarios. Rev. Latino-Am. Enfermagem. 22(5):739746.

9. EL TIEMPO. 2014. Producción de cocaína en el 2013 fue la más baja en 10 años. Periódico El Tiempo, 26 de junio de 2014. Disponible desde Internet en: http://www.eltiempo.com/politica/justicia/baja-produccion-de-cocaina-en-colombia/14175575 (con acceso 17/08/2015).
10. GOBIERNO NACIONAL DE LA REPÚBLICA DE COLOMBIA. 2013. Estudio Nacional de Consumo de Sustancias Psicoactivas en Colombia 2013. Bogotá, 2014. Disponible desde Internet en: https:// www.unodc.org/documents/colombia/2014/Julio/ Estudio_de_Consumo_UNODC.pdf (con acceso $17 / 08 / 2015)$.

11. GONZÁLEZ, Y.; MANN, R.; HAMILTON, H.; ERICKSON, P.; SAPAG, J.; BRANDS, B.; KHENTI, A. 2015. The use of drugs between university student and the relation with abuse during childwood and adolescence. Texto \& Contexto-Enfermagem. 24(spe.):88-96.

12. LONDOÑO PÉREZ, C.; VALENCIA LARA, C. 2008. Asertividad, resistencia a la presión de grupo y consumo de alcohol en universitarios. Acta Col. Psicol. 11(1):155-162.

13. MEDINA, L.; CUNNINGHAM, J.; STRIKE, C.; BRANDS, B.; MIOTTO, M. 2009. Normas percibidas por los estudiantes universitarios acerca de sus pares y el uso de drogas en Bogotá, Colombia. Rev. Latino-Am. Enfermagem. 17(spe):893-899.

14. MEJÍA, O. 2010. ¿Estado y cultura mafiosa en Colombia? Universidad Nacional de Colombia. (Bogotá). 239p.

15. MONTOYA, E.; CUNNINGHAM, J.; BRANDS, B.; STRIKE, C.; WRIGHT, M. 2009. Consumo percibido y uso de drogas lícitas e ilícitas en estudiantes universitarios en la Ciudad de Medellín, Colombia. Rev. Latino-Am. Enfermagem. 17(spe): 886-892.

16. MORALES, B.; PLAZAS, M.; SÁNCHEZ, R.; VENTURA, C. 2011. Factores de riesgo y de protección relacionados con el consumo de sustancias psicoactivas en estudiantes de enfermería. Rev Latino-Am. Enfermagem. 19 (esp):673-683.

17. ORGANIZACIÓN DE LOS ESTADOS AMERICANOS OEA-. 2013. Las drogas en las Américas: Estudios. Capítulo 4. La Economía del Narcotráfico. 47p.

18. ORTEGA-PÉREZ, C.A.; COSTA-JÚNIOR, M.L.D.; VASTERS, G.P. 2011. Perfil epidemiológico de la drogadicción en estudiantes universitarios. Rev. LatinoAm. Enfermagem. 19:665-672.

19. PALMA CAMPOS, C. 2011. Delito y sobrevivencia: las mujeres que ingresan a la cárcel El Buen Pastor en Costa Rica por tráfico de drogas. Anuario Estudios Centroam., U. Costa Rica. 37:245-270. 
20. PRIETO SILVA, R.; SIMICH, L.; STRIKE, C.; BRANDS, B.; GIESBRECHT, N.; KHENTI, A. 2012. Diversidad y complejidad en el fenómeno de las drogas: el policonsumo simultáneo en estudiantes universitarios en una universidad, Cundinamarca - Colombia. Texto Contexto - Enfermería. 21:49-55.

21. RODRÍGUEZ, A.H.; SIMICH, L.; STRIKE, C.; BRANDS, B.; GIESBRECHT, N.; KHENTI, A. 2012. Policonsumo simultáneo de drogas en estudiantes de pregrado del área de la salud en una universidad, León-Nicaragua. Texto \& Contexto Enfermagem. 21:79-86.

22. SECRETARÍA DISTRITAL DE SALUD. 2011. Política pública para la atención y prevención del consumo y la vinculación a la oferta de sustancias psicoactivas en Bogotá D.C.

23. SECRETARÍA GENERAL DE LA COMUNIDAD ANDINA. 2012. II Estudio Epidemiológico Andino sobre Consumo de Drogas en la Población Universitaria. Informe Regional. Perú. Disponible desde Internet en: http://www.cicad.oas.org/oid/pubs/PRADICAN_Informe_Regional.pdf (con acceso 17/08/2015).

24. SEPÚlVEDA, M.; ROA, J.; MUÑOZ, M. 2011. Estudio cuantitativo del consumo de drogas y factores sociodemográficos asociados en estudiantes de una universidad tradicional chilena. Rev. Médica Chile. 139(7):856-863.

25. SIERRA, N. 2012. Drogas y universidad. Consejo Nacional de Control de Sustancias Estupefacientes y Psicotrópicas (Consep). (Quito). Disponible desde Internet: en: http://www.consep.gob.ec/descargas/2014/05/drogas-y-universidad.pdf (con acceso 18/08/2015).

26. TASCON, F. 2012. Cocaína, escenarios de ilegalidad y especulación de capital. América Latina 13/14, Revista del Doctorado en procesos sociales y políticos en América Latina $1^{\circ}$ y $2^{\circ}$ semestre 2012 /Universidad ARCIS. Editorial ARCIS: 197 - 233.

27. THOUMI, F. 2009. Las drogas ilegales, el fracaso de la política antinarcóticos y la necesidad de reformas institucionales en Colombia. En: Toklatian, J. (comp). La guerra contra las drogas en el mundo andino, hacia un cambio de paradigma. Libros del Zorzal. (Buenos Aires). p.51-123.

28. TOMÁS, E.A.; TOMÁS, M.S.A.; SUÁREZ, J.R. 2003. Jóvenes, fin de semana y uso recreativo de drogas: evolución y tendencias del ocio juvenil. Adicciones: 15(2):7-33.

29. UNITED NATIONS OFFICE AND DRUGS AND CRIME -UNODC-. 2012. Informe Mundial sobre las Drogas. New York. Disponible desde Internet en: https://www. unodc.org/documents/dataandanalysis/WDR2012/ Executive_summary_spanish.pdf (con acceso 17/08/2015).

30. UNITED NATIONS OFFICE AND DRUGS AND CRIME -UNODC-. 2013. Informe Mundial sobre las Drogas [Internet]. New York, 2013 Disponible desde Internet en: http://www.unodc.org/documents/lpobrazil//Topics_drugs/WDR/2013/Executive_Summary_translation_-_Spanish_-_V1383706.pdf (con acceso 17/08/2015).

31. VARELA, M.; SALAZAR, I.; CÁCERES, D.; TOVAR, J. 2007. Consumo de sustancias psicoactivas ilegales en jóvenes: factores psicosociales asociados. Pensamiento Psicológico. 3(8):31-45.

Recibido: Noviembre 28 de 2014

Aceptado: Octubre 14 de 2015

\section{Cómo citar:}

Segura, L.S.; Cáliz, N.E. 2015. Consumo de drogas de uso lícito e ilícito en jóvenes universitarios de la U.D.C.A. Rev. U.D.C.A Act. \& Div. Cient. 18(2): 311-319. 